\title{
Variáveis Psicossociais Associadas à Cirurgia Ortognática: Uma Revisão Sistemática da Literatura
}

\author{
Psychosocial Variables Associated with Orthognathic Surgery: \\ A Systematic Literature Review
}

\author{
Sónia Cortinhas Carvalho*, Eugénio Joaquim Martins \& Maria Raquel Barbosa \\ Universidade do Porto, Porto, Portugal
}

\begin{abstract}
Resumo
A cirurgia ortognática é um tipo de tratamento orto-cirúrgico indicado para pacientes com deformidade dentofacial. Este tema tem ganhado repercussão na comunidade científica e, mais recentemente, no ramo das ciências psicológicas, pois as pessoas recorrem cada vez mais a este tipo de tratamento e os psicólogos começam a ser contratados para responderem às necessidades emocionais destes pacientes. Este artigo dedica-se a uma revisão da literatura, dos últimos nove anos, visando explorar particularmente se determinadas características psicossociais do paciente contribuem para a eficácia do tratamento ortocirúrgico. A nossa pesquisa identificou catorze estudos e os resultados são apresentados por setores: (a) fase pré-operatória, (b) fase pós-operatória a curto e médio prazo, (c) fase pós-operatória a longo-prazo. Verificámos que algumas características dos pacientes e uma relação de proximidade estabelecida com os profissionais de saúde parecem contribuir para a satisfação, porém, é apoio psicológico de caráter preventivo dos fatores de risco que parece determinar o sucesso do tratamento orto-cirúrgico. A demasiada valorização das expectativas de benefícios psicológicos, a insegurança na tomada de decisão, o desconhecimento dos custos emocionais da cirurgia, o pobre suporte social (ou a pressão dos familiares) e as perturbações psicopatológicas justificam a utilidade do apoio psicológico. Finalmente são apontadas algumas características psicológicas, positivas e negativas, do paciente assim como sugestões para futuras investigações. Palavras-chave: Cirurgia ortognática, variáveis psicossociais, pré-operatório e pós-operatório.
\end{abstract}

\begin{abstract}
Orthognathic surgery is a type of orthodontic treatment recommended for patients with dentofacial deformities. This issue has grown in the scientific community and, more recently, in the field of psychological science since there is an increasing number of people resorting to this type of treatment and psychologists are being hired to meet emotional needs of such patients. This paper focuses on a review of the literature from the last nine years and seeks to determine if some of the psychosocial characteristics of the patient contribute to the effectiveness of orthodontic-surgical treatment. Our research identified 14 studies and the results are presented according to the following: (a) pre-surgical phase; (b) short-term and mid-term post-surgical phases; and (c) long-term post-surgical phase. We observed that some of the patient's characteristics and a closer patient-caregiver relationship seem to contribute to the satisfaction of the treatment. However, it is the quality of preventive psychological support towards risk factors that seems to determine the success of this orthodontic treatment. Overvaluation of expectations towards psychological benefits, insecurity in the decision making, lack of knowledge about the emotional costs of the surgery, lack of social support (or family pressure) as well as psychopathological disorders justify the need of psychological support. Finally, some positive and negative patient characteristics have been pointed out in this paper, and suggestions for future investigations have been made.

Keywords: Orthognathic surgery, psychosocial variables, pre-surgical and post-surgical.
\end{abstract}

A atratividade facial tem grande importância para as pessoas que procuram o tratamento orto-cirúrgico. Tal como nota Freitas-Magalhães (2007): “O rosto humano é

"Endereço para correspondência: Faculdade de Psicologia e de Ciências da Educação, Universidade do Porto, Rua Alfredo Allen, Porto, Portugal 4200-135. E-mail: soniaacortinhas@gmail.com o palco da nossa identidade e é a parte que mais mostramos aos outros durante toda a vida" (p. 33). Existem, por isso, normas de boa apresentação na interação social que significam permanentes cuidados na aparência física.

$\mathrm{Na}$ verdade, nós criamos expectativas e agimos, muitas vezes, inconscientemente classificando as pessoas em determinadas categorias ou protótipos, e estes pressupostos implícitos mediatizam frequentemente as relações interpessoais (Dion, Berscheid, \& Hatfield, 1972). 
Alguns estudos têm evidenciado que as pessoas com características físicas atraentes tendem a provocar expectativas ou impressões positivas nos outros com a obtenção de vantagens interpessoais. E quando uma pessoa não corresponde aos padrões sociais de beleza física, tende a induzir impressões negativas nos outros, sendolhe exigido melhores resultados e responsabilidades sociais (Belluci \& Kapp-Simon, 2007; Lazaridou-Terzoudi, Kiyak, Athanasiou, \& Melsen, 2003; Phillips, Bennett, \& Broder, 1998). Essa situação é suscetível de provocar um sofrimento psicológico significativo na pessoa, afetando a sua qualidade de vida. Para lidar com o problema dentofacial, ela pode recorrer a estratégias de ocultamento (e.g. cobrir a boca com a mão quando fala, evitar o sorriso, mover os lábios de forma artificial, não gostar de tirar fotografias), ou, em casos extremos, pode manifestar comportamentos de fobia social traduzidos em sentimentos de medo e de insegurança emocional no relacionamento interpessoal.

Estima-se que a deformidade dentofacial afeta aproximadamente $20 \%$ da população mundial (Sadek \& Salem, 2007). Leite et al. (2004) verificaram, numa amostra de 180 pacientes brasileiros portadores da má-oclusão oriundos de um centro de tratamento, que o tipo de deformidade mais submetido ao tratamento foi a Classe III de Angle (53\%). Os tipos de Classe II (33\%) e I ( $2 \%)$ cifraram valores percentuais menos intensos. Gonçalves et al. (2010) averiguaram, numa amostra de 469 pacientes portugueses atendidos num serviço de saúde dentária, a prevalência de má-olusão ligeira na ordem de $46,8 \%$, a má-olusão definitiva correspondia a $26,5 \%$, a má-oclusão severa representava $15,6 \%$, e a má-oclusão muito grave ou inca-pacitante traduzia $11,1 \%$.

A cirurgia ortognática permite, de fato, melhorias na oclusão dentária, na estética facial, aprimorando a função mastigatória e a harmonia da face. As deformidades dentofaciais esqueléticas podem ocorrer em resultado da sífilis congénita, eventos de desenvolvimento ou traumáticos que perturbam o crescimento e o desenvolvimento normal do esqueleto do rosto. A combinação do tratamento ortodôntico e cirúrgico é necessária para restabelecer não só os limites normais do esqueleto do rosto como também para melhorar a função respiratória, motor-oral e a fala, visto que não é possível corrigir a relação entre as arcadas dentárias só com os aparelhos ortodônticos (Belluci \& Kapp-Simon, 2007).

Muitos cirurgiões ortognáticos e ortodontistas já tiveram a experiência de lidar com casos de insatisfação ou de desordens psicopatológicas do paciente, ainda que a sua taxa de incidência varie entre os $5 \%$ e os $24,7 \%$ (C. Phillips et al., 1998; Pogrel \& P. Scott, 1994). Os autores alegam que, nestas circunstâncias, as principais razões estão ligadas às histórias pessoais e necessidades socioafetivas do paciente e não propriamente à aparência facial (por exemplo, submeter-se à cirurgia para salvar ou melhorar uma relação íntima). Outros fatores como o pessi- mismo, a ansiedade, as expectativas irrealistas, o pobre apoio social podem prejudicar, de igual modo, o sucesso do tratamento (Belluci \& Kapp-Simon, 2007; A. Scott et al., 2000). Esses dados apontam-nos para a necessidade de gerenciar as emoções do paciente durante o processo de tratamento, pois o eventual insucesso não se deverá exclusivamente às características psicológicas do paciente, mas sobretudo à deficiente relação de proximidade entre os intervenientes no processo.

A falta de uma relação de proximidade parece prejudicar o paciente, diminuindo a sua motivação para completar as exigências do tratamento ao nível do preparo emocional pré-cirúrgico, assim como ao nível dos cuidados de higiene, dieta alimentar e adaptação emocional pós-cirúrgicos.

No entanto, será importante a investigação nesta área ser ampliada futuramente para uma melhor compreensão das variáveis intervenientes neste processo. Este domínio de investigação é muito recente em Portugal, pelo que ainda não existe uma revisão de literatura sobre o tema. É com o objetivo de preencher esta lacuna na literatura científica que surge este artigo, no entanto, face à diversidade de objetivos específicos para o trabalho, focamo-nos apenas na identificação e análise das características psicossociais do paciente (e.g. estatuto psicológico antes e depois da cirurgia, qualidade de vida, motivos, expectativas, satisfação, etc) que podem estar associadas ao sucesso e/ou insucesso do tratamento ortocirúrgico.

Em suma, podemos dizer que o presente trabalho se dedica a uma revisão das produções científicas no âmbito do tratamento ortognático, atendendo particularmente à seguinte questão: É possível estabelecer uma relação entre as características psicossociais (negativas/positivas) dos pacientes e a eficácia do tratamento orto-cirúrgico (insucesso/ sucesso)?

\section{Método}

Partindo de um artigo de revisão intitulado " $O \mathrm{Im}$ pacto Psicológico da Cirurgia Ortognática: Uma Revisão Sistemática" (Hunt, Johnston, Hepper, \& Burden, 2001), procedeu-se a uma revisão sistemática da literatura sobre o tema supracitado mediante o levantamento de artigos científicos nos últimos nove anos (2001 a 2010), nas seguintes publicações das editoras Elsevier, Oxford University Press e Maney Publishing. Cruzaramse as palavras-chave "cirurgia ortognática" e "paciente ortognático" com "estatuto psicológico", "saúde mental", "avaliação", "qualidade de vida", "satisfação", "tratamento e/ou adesão ao tratamento" e "características psicossociais".

Em todas as bases de dados encontraram-se 297 resultados. A amostra foi refinada, explorando-se os resumos com atenção aos seguintes critérios de inclusão: (a) presença dos termos "pré-operatório" e "pós-operatório", 
Carvalho, S. C., Martins, E. J. \& Barbosa, M. R. (2012). Variáveis Psicossociais Associadas à Cirurgia Ortognática: Uma Revisão Sistemática da Literatura.

uma vez que a discussão dos resultados seguirá esta estrutura tridimensional (por áreas) e (b) modalidade de produção científica, pois selecionamos apenas os estudos empíricos. No total, foram selecionados catorze artigos relevantes para a presente revisão.

A estrutura tridimensional aqui adoptada na apresentação dos resultados (e.g. fase pré-cirúrgica, fase pós-cirúrgica a curto e médio prazo, fase pós-cirúrgica a longoprazo) serve-nos apenas por pragmatismo, porque a parte metodológica de alguns estudos inclui uma linha de base (baseline) que normalmente se inicia na fase précirúrgica quando a intervenção ortodôntica já começou. A linha de base permite-nos comparações de dados entre diferentes momentos localizados no tempo (por exemplo, um mês antes da cirurgia e seis, sete semanas depois da cirurgia).

Como não é possível apresentar uma relação direta entre os fatores de risco inerentes ao paciente e o insucesso do tratamento orto-cirúrgico, tentaremos demonstrar em que medida algumas características psicossociais do paciente (positivas e negativas) e a relação de proximidade estabelecida com o(s) profissional(is) de saúde poderão contribuir, de fato, para o sucesso ou maior insucesso do tratamento.

É possível orientar a revisão neste sentido, uma vez que a avaliação psicológica do paciente que se submete à cirurgia ocorre, normalmente, em função do interesse de analisar a sua capacidade de ajustamento psicossocial e de averiguar se há uma relação entre os seus estados de humor na fase pré-operatória e as suas respostas orgânico-fisiológicas e emocionais na fase pós-operatória (Choi, Lee, McGrath, \& Samman, 2010; Cunningham, Gilthorpe, \& Hunt, 2000; S. Kim, M. Kim, Shin, Chun, \& E. Kim, 2009; Motegi, Hatch, Rugh, \& Yamaguchi, 2003; C. Phillips, Kiyak, Bloomquist, \& Turvey, 2004). Acontece que parecem existir determinados fatores que fazem de alguns pacientes melhores candidatos do que outros.

\section{Resultados}

\section{Características dos Estudos}

A Tabela 1 contém a categorização dos estudos incluídos no presente trabalho. Os desenhos de investigação mais frequentemente utilizados pelos autores são de índole transversal, sem/com grupo de controlo, e de índole prospetivo, sem grupo de controlo. Os menos usados são os desenhos de natureza longitudinal, de subtipo retrospetivo e prospetivo com grupo de controlo.
Tabela 1

Categorização dos Estudos Incluídos na Revisão

Tipo de estudo

a. Desenhos cross-sectional

Transversal sem grupo de controlo

Transversal com grupo de controlo

c. Desenhos de coorte ou longitudinais

Prospetivo com grupo de controlo

Retrospetivo com grupo de controlo

Prospetivo sem grupo de controlo

Retrospetivo sem grupo de controlo

Total de estudos incluídos na revisão

A maioria dos estudos centra-se na caracterização do perfil psicológico do paciente (Burden et al., 2010; Cunningham, Gilthorpe, \& Hunt, 2001; Johnston, Hunt, Burden, Stevenson, \& Hepper, 2010; Juggins, Shute, \& Cunningham, 2006; Stirling et al., 2007; D. Williams et al., 2009), e nas mudanças na sua personalidade além da satisfação pré- e pós-cirúrgicas (Choi et al., 2010; Espeland, Høgevold, \& Stenvik, 2008; S. Kim et al., 2009; Lazaridou-Terzoudi et al., 2003; Motegi et al., 2003; C. Phillips et al., 2004; D. Williams et al., 2009; R. Williams, Travess, \& A. Williams, 2004). A ansiedade, a depressão, o distress, a auto-estima e a imagem corporal correspondem às dimensões psicológicas mais estudadas em detrimento das dimensões aferentes à opinião dos pacientes ou dos profissionais de saúde sobre a utilidade da avaliação psicológica no âmbito do tratamento ortocirúrgico (cf. Tabela 1).

$\mathrm{Na}$ Figura 1 encontram-se identificados os instrumentos de medida utilizados pelos autores nos estudos selecionados para a presente revisão. O questionário é o instrumento privilegiado pelos autores, embora a maioria das medidas não esteja especificamente aferida para a população de candidatos à cirurgia ortognática, à excepção de Orthognathic Quality of Life Questionnaire. Por esta razão, certos autores escolhem utilizar versões que foram testadas em contexto de cirurgia dermatológica ou cosmética, ao passo que outros autores optam, a propósito, por construir questionários correspondentes aos objetivos de investigação.

Os autores escolhem, habitualmente, realizar um préteste, controlando a qualidade da amostra através de critérios de exclusão dos participantes (por exemplo, problemas psiquiátricos, deformidades dentofaciais devido a trauma, síndromes congénitas, realização prévia de cirurgia ortognática) e recolhendo sempre o seu consentimento informado. Também nos grupos de controlo são excluídos colaboradores quando está presente uma deformidade dentofacial. 


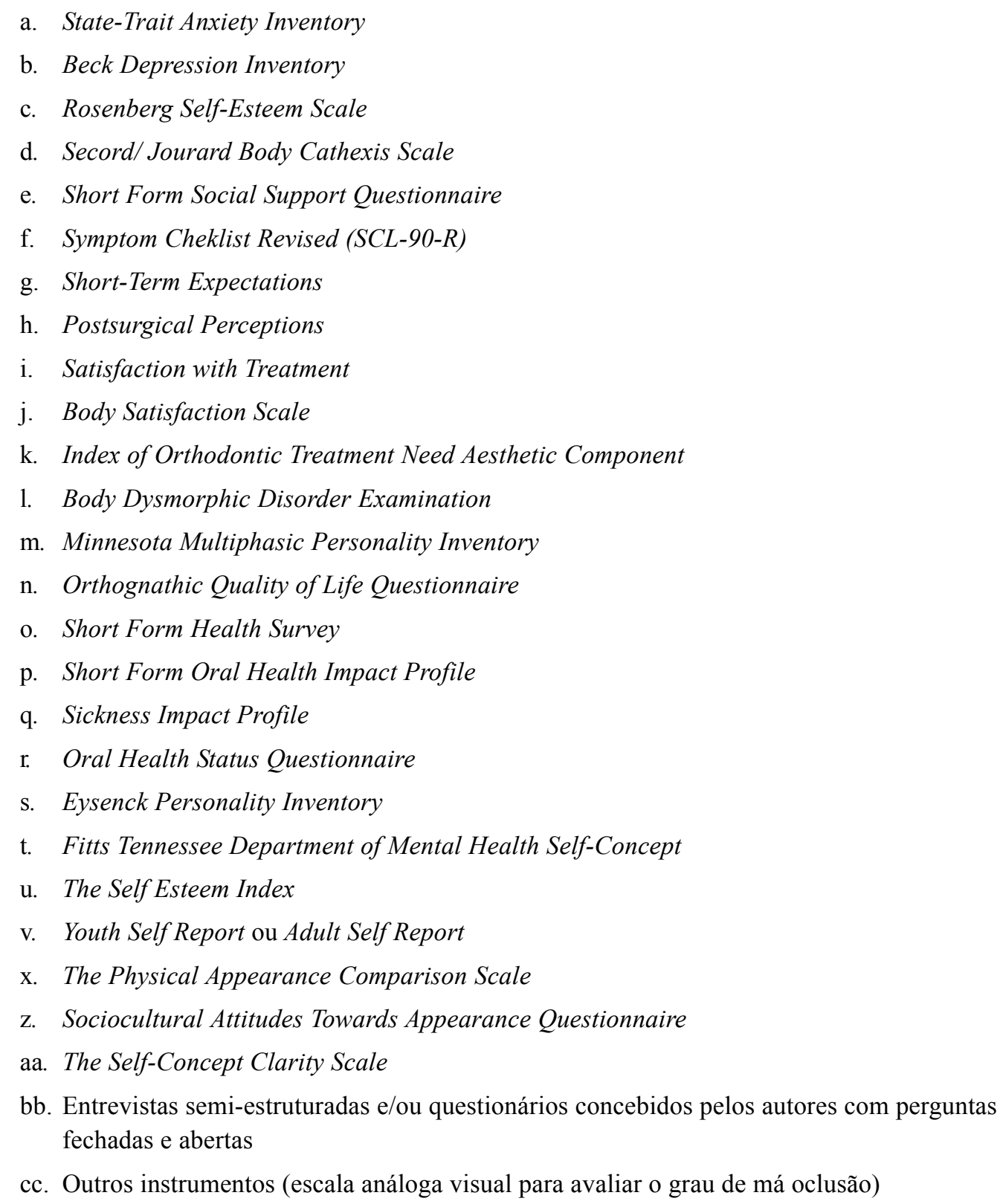

Figura 1. Identificação de questionários usados nos estudos da presente revisão.

A devolução de questionários nunca atinge a taxa de $100 \%$. Alguns participantes desistem de continuar com o tratamento, outros não foram encontrados, a posteriori, para preencher novamente os questionários, outros ainda não terminaram, na fase pós-operatória, o tratamento ortodôntico durante o período de recolha dos dados.
A Tabela 2 descreve resumidamente as características dos estudos selecionados para o presente trabalho de revisão, mencionando-se, entre outros aspetos, o tipo de investigação, os objetivos, as dimensões psicológicas analisadas e os seus principais resultados. Posteriormente, será efetuada uma discussão do conteúdo dos artigos, particularmente dos seus resultados. 
Carvalho, S. C., Martins, E. J. \& Barbosa, M. R. (2012). Variáveis Psicossociais Associadas à Cirurgia Ortognática: Uma Revisão Sistemática da Literatura.

Tabela 2

Organização Sistemática dos Estudos Incluídos na Presente Revisão

\begin{tabular}{|c|c|c|c|c|c|c|}
\hline $\begin{array}{l}\text { Tipo de } \\
\text { estudo }\end{array}$ & $\begin{array}{l}\text { Tamanho } \\
\text { da } \\
\text { amostra }\end{array}$ & Baseline & Follow-up & Objetivos & $\begin{array}{c}\text { Identificação } \\
\text { de } \\
\text { questionários }\end{array}$ & $\begin{array}{c}\text { Principais resultados/ } \\
\text { conclusões }\end{array}$ \\
\hline
\end{tabular}

TRANSVERSAL SEM GRUPO DE CONTROLO

\begin{tabular}{|c|c|c|c|c|c|c|}
\hline $\begin{array}{l}\text { Juggins } \\
\text { et al. } \\
\text { (2006) }\end{array}$ & 177 & - & - & $\begin{array}{l}\text { avaliar a } \\
\text { opinião sobre } \\
\text { a utilidade } \\
\text { da avaliação } \\
\text { psicológica } \\
\text { junto dos } \\
\text { ortodontistas }\end{array}$ & $\mathrm{bb}$ & $\begin{array}{l}\text { - aproximadamente } 40 \% \text { dos ortodontistas } \\
\text { consideravam a necessidade de avaliação } \\
\text { psicológica para } 10 \% \text { de todos os pacien- } \\
\text { tes que escolhem o tratamento orto-cirúr- } \\
\text { gico, enquanto } 12 \% \text { entendiam que todos } \\
\text { os candidatos deveriam submetidos à ava- } \\
\text { liação antes de qualquer tratamento ativo. }\end{array}$ \\
\hline $\begin{array}{l}\text { Vulink } \\
\text { et al. } \\
\text { (2008) }\end{array}$ & 160 & & & $\begin{array}{l}\text { determinar a } \\
\text { prevalência } \\
\text { da Perturbação } \\
\text { Dismórfica } \\
\text { Corporal }\end{array}$ & 1 & $\begin{array}{l}\text { - cerca de } 17 \% \text { dos candidatos tinham uma } \\
\text { preocupação excessiva com a sua aparên- } \\
\text { cia, induzindo-lhes stresse prejudicial e } \\
\text { funcionamento psicossocial disruptivo; } \\
\text { desses candidatos, } 10 \% \text { preenchiam os cri- } \\
\text { térios clínicos da desordem psiquiátrica. }\end{array}$ \\
\hline $\begin{array}{l}\text { Stirling } \\
\text { et al. } \\
\text { (2007) }\end{array}$ & 61 & - & - & $\begin{array}{l}\text { explorar a } \\
\text { decisão do } \\
\text { ponto de vista } \\
\text { dos pacientes } \\
\text { e o seu estatuto } \\
\text { psicológico em } \\
\text { termos de ansie- } \\
\text { dade, auto-estima } \\
\text { e satisfação } \\
\text { corporal }\end{array}$ & $\mathrm{a}, \mathrm{c}, \mathrm{j}, \mathrm{cc}$ & $\begin{array}{l}\text { - os pacientes pareciam tomar decisões } \\
\text { baseadas em pouca informação sobre os ris- } \\
\text { cos e benefícios do tratamento orto-cirúr- } \\
\text { gico, inclusive nas suas necessidades emo- } \\
\text { cionais; } \\
\text { - para estes, a provisão de informações so- } \\
\text { bre a situação da cirurgia não foi totalmente } \\
\text { satisfatória; } \\
\text { - os pacientes não diferiam significativa- } \\
\text { mente da população geral quanto ao esta- } \\
\text { tuto psicológico. }\end{array}$ \\
\hline
\end{tabular}

\section{TRANSVERSAL COM GRUPO DE CONTROLO}

\begin{tabular}{|c|c|c|c|c|c|c|}
\hline $\begin{array}{l}\text { D. Williams } \\
\text { et al. } \\
(2009)\end{array}$ & $\begin{array}{l}30 \text { g.p } \\
30 \text { g.c }\end{array}$ & - & - & $\begin{array}{l}\text { analisar a } \\
\text { satisfação com a } \\
\text { imagem corporal, } \\
\text { o auto-conceito, } \\
\text { a depressão, } \\
\text { ansiedade e a } \\
\text { auto-estima }\end{array}$ & $\begin{array}{c}\mathrm{c}, \mathrm{x}, \mathrm{j}, \mathrm{z} \\
\mathrm{aa}, \mathrm{bb}\end{array}$ & $\begin{array}{l}\text { - os candidatos (género feminino) tinham } \\
\text { um funcionamento psicológico normal, } \\
\text { mas mostravam significativamente mais in- } \\
\text { satisfação com a sua aparência e elevada } \\
\text { ansiedade. }\end{array}$ \\
\hline $\begin{array}{l}\text { Burden } \\
\text { et al. } \\
(2010)\end{array}$ & $\begin{array}{l}162 \text { g.p } \\
157 \text { g.c }\end{array}$ & - & - & $\begin{array}{l}\text { examinar o } \\
\text { estatuto psico- } \\
\text { lógico ao nível } \\
\text { da ansiedade, } \\
\text { depressão, auto- } \\
\text { estima e desordens } \\
\text { comportamentais }\end{array}$ & $\mathrm{u}, \mathrm{v}$ & $\begin{array}{l}\text { - não houve diferenças significativas, sen- } \\
\text { do que os candidatos à cirurgia apresenta- } \\
\text { vam um bom funcionamento psicológico } \\
\text { apesar de mostrarem tendencialmente pio- } \\
\text { res resultados nas dimensões psicológicas } \\
\text { supra-referidas. }\end{array}$ \\
\hline $\begin{array}{l}\text { Johnston } \\
\text { et al. } \\
(2010)\end{array}$ & $\begin{array}{l}162 \text { g.p } \\
157 \text { g.c }\end{array}$ & - & - & $\begin{array}{l}\text { avaliar a } \\
\text { perceção } \\
\text { da atratividade } \\
\text { facial e dentária }\end{array}$ & $\mathrm{k}, \mathrm{cc}$ & $\begin{array}{l}\text { - a presença da deformidade dentofacial } \\
\text { contribuía para a predição da perceção da } \\
\text { atratividade, sendo que os candidatos } \\
\text { mostravam-se pouco felizes com a sua } \\
\text { aparência; } \\
\text { - aproximadamente } 96 \% \text { dos candidatos } \\
\text { desejavam mudar a sua aparência. }\end{array}$ \\
\hline
\end{tabular}




\begin{tabular}{|c|c|c|c|c|c|c|}
\hline $\begin{array}{l}\text { Tipo de } \\
\text { estudo }\end{array}$ & $\begin{array}{c}\text { Tamanho } \\
\text { da } \\
\text { amostra }\end{array}$ & Baseline & Follow-up & Objetivos & $\begin{array}{l}\text { Identificação } \\
\text { de } \\
\text { questionários }\end{array}$ & $\begin{array}{l}\text { Principais resultados/ } \\
\text { conclusões }\end{array}$ \\
\hline
\end{tabular}

\section{PROSPETIVO SEM GRUPO DE CONTROLO}

\begin{tabular}{|c|c|c|c|c|c|c|}
\hline $\begin{array}{l}\text { Choi } \\
\text { et al. } \\
(2010)\end{array}$ & 32 & $\begin{array}{l}\text { 1) no recru- } \\
\text { tamento }\end{array}$ & $\begin{array}{l}\text { 2) no pós- } \\
\text { operatório de } \\
\text { seis semanas, } \\
\text { 3) no pós- } \\
\text { operatório de } \\
\text { seis meses, e } \\
\text { 4) no fim da } \\
\text { intervenção } \\
\text { ortodôntica } \\
\text { (seis a doze } \\
\text { meses) }\end{array}$ & $\begin{array}{l}\text { determinar } \\
\text { as mudanças } \\
\text { na qualidade } \\
\text { de vida }\end{array}$ & $\mathrm{n}, \mathrm{o}, \mathrm{p}$ & $\begin{array}{l}\text { - houve mudanças significativas, em con- } \\
\text { creto, a deterioração da qualidade de vida } \\
\text { no pós-operatório de seis semanas que foi } \\
\text { originada pelos sintomas de dor e limita- } \\
\text { ções funcionais; } \\
\text { - as melhorias no bem-estar pessoal surgi- } \\
\text { ram particularmente entre o pós-operató- } \\
\text { rio de seis semanas e o pós-operatório de } \\
\text { seis meses, estabilizando-se na fase final } \\
\text { do tratamento. }\end{array}$ \\
\hline $\begin{array}{l}\text { Motegi } \\
\text { et al. } \\
(2003)\end{array}$ & 93 & $\begin{array}{l}\text { 1) uma } \\
\text { a duas } \\
\text { semanas } \\
\text { antes da } \\
\text { cirurgia }\end{array}$ & $\begin{array}{l}\text { 2) no pós- } \\
\text { operatório } \\
\text { de dois anos, } \\
\text { e } 3 \text { ) no pós- } \\
\text { operatório } \\
\text { de cinco } \\
\text { anos }\end{array}$ & $\begin{array}{l}\text { avaliar o } \\
\text { estado de } \\
\text { saúde oral } \\
\text { e mental }\end{array}$ & $\mathrm{q}, \mathrm{r}, \mathrm{s}, \mathrm{f}$ & $\begin{array}{l}\text { - as mudanças significativas refletiram-se } \\
\text { na melhoria dos relacionamentos interpes- } \\
\text { soais, comunicação, satisfação com a ima- } \\
\text { gem e estabilidade emocional, mantendo-se } \\
\text { estáveis até cinco anos depois da cirurgia; } \\
\text { - a evolução foi mais pronunciada entre o } \\
\text { pré-operatório e a pós-operatório de dois } \\
\text { anos, mas estabilizou-se até cinco anos de- } \\
\text { pois da cirurgia. }\end{array}$ \\
\hline
\end{tabular}

Cunnin- $62 \quad 1$ ) início da gham et al. (2001)

\section{2) fim da preparação ortodôntica}

identificar as mudanças no estatuto psicológico em termos de ansiedade, depressão, auto-estima, imagem corporal e imagem facial.

analisar o estatuto psicológico, expectativas pré-cirúrgicas, perceções pós-cirúrgicas e satisfação $\mathrm{a}, \mathrm{b}, \mathrm{c}, \mathrm{d}, \quad$ - a imagem corporal foi a única dimensão e, cc psicológica afetada significativamente pela intervenção ortodôntica.

\begin{tabular}{|c|c|c|c|c|}
\hline $\begin{array}{l}\text { C. } \\
\text { Phillips } \\
\text { et al. } \\
(2004)\end{array}$ & 126 & $\begin{array}{l}\text { 1) um } \\
\text { a dois } \\
\text { meses } \\
\text { antes da } \\
\text { cirurgia }\end{array}$ & $\begin{array}{l}\text { 2) após } \\
\text { quatro a } \\
\text { seis } \\
\text { semanas } \\
\text { do ato } \\
\text { cirúrgico }\end{array}$ & $\begin{array}{l}\text { analisar o } \\
\text { estatuto } \\
\text { psicológico, } \\
\text { expectativas } \\
\text { pré-cirúrgicas, } \\
\text { perceções } \\
\text { pós-cirúrgicas } \\
\text { e satisfação }\end{array}$ \\
\hline
\end{tabular}

$\mathrm{f}, \mathrm{g}, \mathrm{h}, \mathrm{i}$ - assistir à simulação, antes da cirurgia, não prejudica significativamente o bem-estar dos pacientes, mas a simulação é útil quando eles mostram sintomas severos de stresse;

- aqueles que, inicialmente, sobrestimaram o desconforto do tratamento, acabaram por admitir, de forma significativa, menos problemas pós-cirúrgicos em comparação com aqueles que subestimaram o desconforto.

\section{PROSPETIVO COM GRUPO DE CONTROLO}

\begin{tabular}{|c|c|c|c|c|c|c|}
\hline $\begin{array}{l}\text { S. Kim } \\
\text { et al. } \\
(2009)\end{array}$ & $\begin{array}{l}34 \text { g.p } \\
30 \text { g.c }\end{array}$ & $\begin{array}{c}\text { 1) antes } \\
\text { da cirurgia }\end{array}$ & $\begin{array}{l}\text { 2) no pós- } \\
\text { operatório de } \\
\text { sete semanas } \\
\text { e 3) no pós- } \\
\text { operatório } \\
\text { de seis meses }\end{array}$ & $\begin{array}{l}\text { avaliar o } \\
\text { estatuto } \\
\text { psicológico e } \\
\text { características } \\
\text { de } \\
\text { personalidade }\end{array}$ & $\mathrm{m}, \mathrm{f}$ & $\begin{array}{l}\text { - os candidatos à cirurgia tinham bom fun- } \\
\text { cionamento psicológico, apesar de uma } \\
\text { personalidade tendencialmente defensiva; } \\
\text { - no pós-operatório de seis meses, houve } \\
\text { melhorias significativas nos sintomas de } \\
\text { depressão, hipocondria e paranóia. }\end{array}$ \\
\hline
\end{tabular}


Carvalho, S. C., Martins, E. J. \& Barbosa, M. R. (2012). Variáveis Psicossociais Associadas à Cirurgia Ortognática: Uma Revisão Sistemática da Literatura.

\begin{tabular}{|c|c|c|c|c|c|c|}
\hline $\begin{array}{l}\text { Tipo de } \\
\text { estudo }\end{array}$ & $\begin{array}{l}\text { Tamanho } \\
\text { da } \\
\text { amostra }\end{array}$ & Baseline & Follow-up & Objetivos & $\begin{array}{c}\text { Identificação } \\
\text { de } \\
\text { questionários }\end{array}$ & $\begin{array}{c}\text { Principais resultados/ } \\
\text { conclusões }\end{array}$ \\
\hline
\end{tabular}

\section{RETROSPETIVO COM GRUPO DE CONTROLO}

\begin{tabular}{|c|c|c|c|c|c|}
\hline $\begin{array}{l}\text { Lazaridou- } \\
\text { Terzoudi } \\
\text { et al. } \\
(2003)\end{array}$ & $\begin{array}{l}117 \text { g.p } \\
39 \text { g.c } \\
92 \text { g.c }\end{array}$ & $\begin{array}{l}\text { 1) pacientes } \\
\text { que realizaram } \\
\text { a cirurgia } \\
\text { entre } 1982 \\
\text { a } 1986 \\
\text { participaram } \\
\text { pouco tempo } \\
\text { depois num } \\
\text { estudo } \\
\text { retrospetivo }\end{array}$ & $\begin{array}{l}\text { 2) dez a } \\
\text { catorze } \\
\text { anos após } \\
\text { a cirurgia }\end{array}$ & $\begin{array}{l}\text { explorar a d, t, bb } \\
\text { perceção } \\
\text { de problemas } \\
\text { quanto à } \\
\text { função oral, } \\
\text { saúde geral, } \\
\text { imagem } \\
\text { corporal } \\
\text { e relações } \\
\text { interpessoais }\end{array}$ & $\begin{array}{l}\text { - os pacientes reportavam significati- } \\
\text { vamente melhores níveis de saúde, fun- } \\
\text { ção oral, bem-estar interpessoal e sa- } \\
\text { tisfação com o perfil facial do que os } \\
\text { candidatos, mas não existiram diferen- } \\
\text { ças significativas em relação aos sujei- } \\
\text { tos saudáveis. }\end{array}$ \\
\hline
\end{tabular}

\section{RETROSPETIVO SEM GRUPO DE CONTROLO}

\begin{tabular}{|c|c|c|c|c|c|c|}
\hline $\begin{array}{l}\text { R. Williams } \\
\text { et al. } \\
(2004)\end{array}$ & 327 & - & $\begin{array}{l}\text { 1) dois } \\
\text { anos após } \\
\text { a cirurgia }\end{array}$ & $\begin{array}{l}\text { explorar a } \\
\text { opinião sobre } \\
\text { a experiência } \\
\text { do tratamento } \\
\text { orto-cirúrgico }\end{array}$ & $\mathrm{bb}$ & $\begin{array}{l}\text {-cerca de } 50 \% \text { dos pacientes conside- } \\
\text { ravam-se muito satisfeitos com a mu- } \\
\text { dança facial; } \\
\text { - a sintomatologia clínica no pós-ope- } \\
\text { ratório imediato foi pior do que tinham } \\
\text { antecipado (e.g. dores - } 32 \% \text {, inchaço } \\
\text { - } 51 \% \text {, dificuldade em comer - } 17 \% \text { e } \\
\text { em respirar-31\%). }\end{array}$ \\
\hline $\begin{array}{l}\text { Espeland } \\
\text { et al. } \\
(2008)\end{array}$ & 516 & - & $\begin{array}{l}\text { 1) três } \\
\text { anos após } \\
\text { a cirurgia }\end{array}$ & $\begin{array}{l}\text { analisar a } \\
\text { opinião e a } \\
\text { satisfação } \\
\text { com os } \\
\text { resultados } \\
\text { cirúrgicos }\end{array}$ & $\mathrm{bb}$ & $\begin{array}{l}\text { - a maioria dos pacientes mostrava-se } \\
\text { satisfeita com o resultado da cirurgia } \\
(92 \%) \text { em termos de reparação da ca- } \\
\text { pacidade de oclusão dentária e atrativi- } \\
\text { dade facial; } \\
\text {-globalmente, } 36.8 \% \text { denunciavam a di- } \\
\text { minuição da função sensorial como sen- } \\
\text { do o efeito colateral mais frequente. }\end{array}$ \\
\hline
\end{tabular}

Nota. G.P (Grupo de Pacientes); G.C (Grupo de Controlo).

\section{A Revisão}

Fase Pré-Operatória. A preocupação com a estética facial tem sido o principal motivo apontado pelos pacientes para justificarem o tratamento orto-cirúrgico (Belluci \& Kapp-Simon, 2007; Hunt et al., 2001; Jacobson, 1984; Johnston et al., 2010; Pogrel \& P. Scott, 1994; Sadek \& Salem, 2007; Stirling et al., 2007; Vulink et al., 2008). De fato, a aparência facial constitui "uma motivação muito importante para que o paciente procure o tratamento ortocirúrgico, pois a beleza, em nossa sociedade, é muito valorizada e é um fator determinante no próprio relacionamento com as pessoas" (Ribas, Reis, França, \& Lima, 2005, p. 76).

Por outro lado, o fato de a estética facial se constituir como o principal motivo do tratamento estará relacionado com as necessidades afetivas do paciente (e.g. de ser capaz de auto-apreciar, de querer ser valorizado(a) pelos outros, de sentir-se confortável no contato social). Estas parecem influenciar, de modo direto, a tomada de deci- são independentemente do nível de conhecimento que o paciente tenha sobre o tratamento (Stirling et al., 2007). Assim, entendemos que o desejo de melhorar a aparência é acompanhado por expectativas de benefícios psicológicos em termos de melhoria da qualidade de vida, mas é preciso reconhecer que, embora as expectativas positivas sejam uma componente importante na motivação do paciente, não preparam para lidar com as circunstâncias imediatas do pós-operatório. A demasiada valorização dos benefícios, em detrimento do conhecimento das implicações reais do processo de tratamento, pode provocar ilusões e consequente insatisfação.

Os casos de insatisfação, embora não sejam proeminentes, variam entre os $13 \%$ e os $25 \%$ (Finlay, Atkinson, \& Moos, 1995; S. Kim et al., 2009; Nicodemo, Pereira, \& Ferreira, 2007; Turker, Varol, \& Basa, 2008; Zane, Tavano, \& Peres, 2002). De acordo com o estudo de Stirling et al., a insatisfação é atribuída aos seguintes fatores: (a) desconhecimento dos riscos colaterais da ci- 
rurgia (por exemplo, falta de sensibilidade no nervo mental), (b) necessidade de mais informações para lidar psicologicamente com os custos emocionais da cirurgia, e (c) necessidade de suporte emocional (preferencialmente prestado por alguém que fez a cirurgia) para lidar com as preocupações acerca do procedimento cirúrgico.

A intervenção ortodôntica pode acentuar os estados emocionais negativos do paciente em consequência das mudanças na sua aparência facial e dentária. De entre as dimensões psicológicas afetadas pela intervenção ortodôntica destaca-se a imagem corporal, conforme o estudo de Cunningham et al. (2001). Por seu lado, os níveis de ansiedade, de depressão e de auto-estima não tendem a sofrer alterações significativas na fase pré-operatória, i.e., antes de qualquer tratamento ativo e no fim da preparação ortodôntica. Portanto, a forma como o paciente percebe a sua imagem facial e corporal poderá ter repercussões evidentes no seu bem-estar, pois a estética facial influencia a formação da personalidade e as características pessoais (e.g. auto-conceito). De fato, a imagem corporal é essencial para o nosso auto-conceito e tem importantes implicações em múltiplas áreas de funcionamento psicológico e na nossa qualidade de vida (Barbosa, 2008).

Os pacientes com deformidade dentofacial tendem a demonstrar altos níveis de ansiedade, reduzida satisfação com a imagem facial e dentária, reduzida auto-estima, sentimentos inseguros quanto à sua feminilidade ou masculinidade, sintomas depressivos e personalidade defensiva (Burden et al., 2010; Cunningham et al., 2000; Honigman, Phillips, \& Castle, 2004; S. Kim et al., 2009; C. Phillips et al., 1998; D. Williams et al., 2009; R. Williams et al., 2004). A nosso ver, essas características fazem parte de um estado transitório e, eventualmente, reativo ao processo de tratamento, cujos parâmetros psicológicos começam a modificar-se depois da cirurgia até ao fim do tratamento. Porquanto, essas características serão indicadores do sofrimento da pessoa, em vez de constituírem fundamentos únicos de diagnóstico psiquiátrico.

Por vezes, o sofrimento pode ser tão acentuado a ponto de o paciente não conseguir geri-lo de forma apropriada e, por conseguinte, elevados níveis de emocionalidade negativa estimulam o aparecimento de distúrbios psicopatológicos ligados à imagem corporal. Vulink et al. (2008) inferiram que $17 \%$ dos candidatos tinham uma preocupação excessiva com a sua aparência, induzindolhes stresse prejudicial e funcionamento psicossocial disruptivo. Desses candidatos, $10 \%$ preenchiam critérios clínicos da Perturbação Dismórfica Corporal que se caracteriza por preocupações excessivas com a aparência, sendo que esta foi mais frequente no grupo de candidatos com Classe II de Angle. Outros autores acrescentam a perturbação obsessiva-compulsiva, o stresse, a hipocondria e a ideação suicida (Belluci \& Kapp-Simon, 2007; Burden et al., 2010; Cunningham \& Feinmann, 1998; S. Kim et al., 2009; A. Scott et al., 2000; Turker et al., 2008). Estes distúrbios aparecem associados às queixas de dor e à insatisfação com os eventos imediatos do pós-operatório (Panula, Finne, \& Oikarinen, 2001).

Relativamente ao grau de disfunção esquelética, a literatura aponta que os candidatos com Classe II de Angle tendem a exibir mais a Perturbação Dismórfica Corporal (Vulink et al., 2008), são os menos felizes com a sua aparência dentária (Johnston et al., 2010), tendem a mostrar reduzida auto-estima, sintomas depressivos, ansiedade e hostilidade (Burden et al, 2010), enquanto os candidatos com Classe III de Angle relatam mais preocupações, insegurança e consciência acerca do seu perfil facial (Johnston et al., 2010). Neste mister, parece-nos que aqueles que têm a deformidade com Classe III estão mais preparados para a mudança facial do que aqueles que têm a Classe II, já que aceitam melhor a ideia da transformação facial e são menos exigentes quanto ao resultado cirúrgico.

Na nossa ótica, os procedimentos de avaliação psicológica não devem visar propósitos de seleção dos melhores candidatos, mas sim servir para identificar perceções erróneas e características da personalidade do paciente que podem perturbar a recuperação pós-cirúrgica. Importa uma boa preparação do paciente para gerir os custos emocionais da cirurgia, já que se trata de um ato bastante invasivo feito na zona mais exposta do corpo. De realçar, entretanto, que não há dados empíricos consistentes que apontem os transtornos psiquiátricos como uma contraindicação absoluta para a realização da cirurgia ortognática. No entanto, a identificação de tais perturbações não deixa de ser valorativa, porque se um transtorno é detetado, os planos de tratamento devem ser ajustados às especificidades do caso.

As expectativas irrealistas, a realização prévia de cirurgia cosmética, a insegurança na tomada de decisão, o pobre apoio social (ou a pressão da família), o uso de substâncias psicotrópicas e a ausência de problema clínico justificativo da cirurgia são outras razões para se referir à utilidade da avaliação psicológica.

A inclusão dos profissionais de saúde mental em equipas que providenciam o serviço de tratamento orto-cirúrgico quer em contexto hospitalar, quer em contexto da clínica de medicina dentária, afigura-se útil. Nesta perspetiva, Juggins et al. (2006) afirmam que $40 \%$ dos ortodontistas sentem a necessidade de beneficiar de parcerias com terapeutas aptos a responder às necessidades emocionais dos pacientes, mas alguns obstáculos impedem o encaminhamento dos pacientes para esses profissionais: (a) desconhecimento de terapeutas especialistas recomendados, (b) receio de uma má reação do paciente, (c) longa lista de espera e (d) pouco aproveitamento da informação exposta nos relatórios clínicos. Como alternativa, alguns ortodontistas optam por pedir aos pacientes, o preenchimento de questionários clínicos referenciados na literatura, no entanto, a maioria não o faz por falta de formação específica na área da psicologia. Os autores sugerem que o paciente deve compreender que o acompanhamento psicológico é uma parte normal do tratamento. 
Seria preferível que um psiquiatra ou um psicólogo estivesse presente na clínica ou no hospital onde são feitos os atendimentos, mas sabemos que, por vezes, isso não é possível. Então o paciente pode ser informado de que seria benéfico para si consultar um membro da equipa noutro local para que possa discutir as suas preocupações e expectativas, ou até procurar apoio para a sua tomada de decisão.

De assinalar, porém, que há poucos estudos publicados sobre as opiniões dos pacientes quanto à utilidade do acompanhamento psicológico neste processo (Juggins et al., 2006; Ryan, Shute, \& Cunningham, 2009), e não existem estudos sobre os procedimentos ou estratégias úteis de aconselhamento psicológico que são usados no contexto de tratamento orto-cirúrgico. A maior parte da produção científica centra-se na caracterização do estatuto psicossocial e da qualidade de vida dos pacientes, antes e depois da cirurgia ortognática.

Fase Pós-Operatória (a curto e médio-prazo). O período pós-operatório imediato corresponde à fase mais exigente do processo de tratamento. O sofrimento psíquico que a pessoa tem vindo a suportar ao longo da sua vida culmina no período pós-operatório a curto-prazo, pelo que a falta de preparação psicológica para lidar com os efeitos da cirurgia pode atrasar a recuperação póscirúrgica e, com efeito, diminuir o sucesso do tratamento, traduzido, em parte, na insatisfação/ desmotivação do paciente.

Os sintomas mais significativos desta fase abrangem a fraqueza física, as insónias, os problemas de dieta alimentar, a parestesia, o inchaço facial, a perda ou ganho de peso, a dificuldade de comunicação oral e o isolamento resultante da restrição de atividades socioprofissionais (Silva, 2009).

A deterioração da qualidade de vida originada pela dor e limitações funcionais acontece, sobretudo, nas primeiras seis semanas a três meses (Lee, McGrath, \& Samman, 2008; Finlay et al., 1995; C. Phillips et al., 1998; Stewart \& Sexton, 1987). Esta será tanto mais grave quanto menos realistas forem as expectativas sobre a sintomatologia do pós-operatório imediato. Isso quer dizer que a recuperação será fortemente determinada pelo nível de conhecimento que se tem do processo de recuperação e como são antecipadas as consequências emocionais. C. Phillips et al. (1998) observaram que os pacientes que inicialmente "sobreestimaram" o desconforto e a dor na fase pós-cirúrgica acabaram por admitir menos problemas em lidar com os sintomas (69\%), em contraste com aqueles que tinham "subestimado" os custos emocionais da cirurgia (31\%). Porquanto, a antecipação (ou as expectativas) dos efeitos da cirurgia influencia a intensidade dos sintomas que são experienciados durante a recuperação.

Do exposto, podemos entender como é que a falta de informação específica sobre a situação da cirurgia pode prejudicar o paciente, aumentando o seu sofrimento. Por este motivo, será importante os profissionais de saúde darem informações corretas e realistas, pois além de mostrar os riscos e os benefícios, não são criadas ilusões (Ribas et al., 2005). R. Williams et al. (2004) averiguaram que cerca de $24 \%$ dos candidatos não se consideravam bem informados sobre o que esperar no pós-operatório imediato e nos cuidados a ter depois da alta hospitalar. Noutro estudo semelhante, Finlay et al. (1995) apuraram que, no pós-operatório de três meses, $42 \%$ dos pacientes denunciavam elevados níveis de neuroticismo, sintomas de dor, de inchaço e de dormência em relação ao esperado e queixavam-se de que receberam pouca informação sobre a situação da cirurgia. Neste sentido, deduzimos que as orientações apropriadas dadas aos pacientes podem contribuir para relacionamentos adequados e positivos com os profissionais de saúde, aumentando a confiança quanto ao sucesso do tratamento.

Qualquer situação desconhecida é suscetível de gerar insatisfação com o resultado da cirurgia e atitudes negativas para com os profissionais de saúde. A médio-prazo, o processo de recuperação e reabilitação ortodôntica poderá complicar-se face à diminuição da motivação do paciente para completar as exigências dos cuidados no pós-operatório. A longo-prazo, os comportamentos negativos poderão coibir o ajustamento à nova aparência facial e a satisfação com o tratamento (Espeland et al., 2008; Ribas et al., 2005; Zane et al., 2002).

Quando o paciente ostenta alguma perturbação psicológica, os sintomas podem ganhar expressão severa na fase pós-cirúrgica. Por exemplo, se o paciente é propenso à depressão, o trauma da cirurgia exacerba a condição ou precipita um grave episódio depressivo. Aliás, a grande variação dos estados emocionais e físicos que caracterizam este período potenciam sintomas depressivos com alterações no seu estado psico-orgânico e nas suas respostas imunológicas. Por esta razão, é sugerido que a depressão constitui um estado reativo aos resultados imediatos da cirurgia ao invés de ser um estado endógeneo (Cunningham et al., 2000).

O stresse exibido por alguns pacientes pode ser minorado com a preparação psicológica providenciada por terapeutas aptos a responder às suas necessidades emocionais. A literatura tem evidenciado que os pacientes que demonstram distress antes da cirurgia acabam por denunciar mais desconforto e preocupações com a saúde até seis semanas depois da cirurgia (C. Phillips et al., 1998), e que o distress está positivamente correlacionado com a insatisfação referente à falta de preparação psicológica comparativamente à insatisfação global com os resultados (Hatch et al., 1999).

Por seu lado, é aceite que o tratamento potencia transformações positivas na saúde física, emocional e nas relações interpessoais: "A relação maxilo-mandibular corrigida favorecerá a função mastigatória, a fonética, a respiração e a estética facial. Porquanto, muitas implicações estão envolvidas neste tratamento cirúrgico, pois a mudança facial repercute-se na vida pessoal e social do indivíduo" (Ribas et al., 2005, p. 75). 
De entre as dimensões psicológicas estudadas pelos autores, é muito provável que a imagem corporal se modifique mais depressa do que a auto-estima, a qualidade de vida, a satisfação, etc. Em consonância com esta perspetiva, os estudos longitudinais têm apontado que a melhoria da imagem corporal já se nota seis semanas (Gerzanic, Jagsch, \&Watzke, 2002) e quatro meses após a cirurgia (Kiyak, McNell, \& West, 1985). As mudanças na qualidade de vida sobressaem particularmente entre o período pós-operatório de seis semanas e de seis meses, estabilizando-se na fase final do tratamento (seis a doze meses; Choi et al., 2010; Lee et al., 2008). Ao fim de seis meses, têm-se observado melhorias no funcionamento psicológico dos pacientes no que diz respeito aos sintomas de fadiga, depressão, hipocondria, paranóia, hostilidade e fobia social (Hatch et al., 1999; S. Kim et al., 2009; Kiyak et al., 1985).

Todavia, não há dados consistentes relativamente ao período exato (pós-cirúrgico), durante o qual os benefícios psicológicos começam a surgir, por causa da ausência de um critério fixo quanto aos limites dos períodos de baseline e follow-up adequados para proceder à avaliação do estatuto psicológico de pacientes. A comprová-lo está o fato de estes serem avaliados, na fase pré-cirúrgica, no início da intervenção ortodôntica (Cunningham et al., 2001), um mês/dois meses (Finlay et al., 1995; C. Phillips et al., 2004), uma/duas semanas (Motegi et al., 2003) ou até poucos dias antes da cirurgia (S. Kim et al., 2009). Denota-se, entretanto, maior variabilidade nos momentos de avaliação na fase pós-cirúrgica - quatro/ seis semanas (Choi et al., 2010; C. Phillips et al., 2004), sete semanas (S. Kim et al., 2009), seis/ doze meses (Choi et al., 2010; Finlay et al., 1995; S. Kim et al., 2009), dois/ cinco anos (Motegi et al., 2003; A. Scott et al., 1999), três anos (Espeland et al., 2008) e dez/catorze anos depois da cirurgia (Lazaridou-Terzoudi et al., 2003).

Desses estudos, averiguámos que quando é feita a avaliação do estatuto psicológico dos pacientes poucos dias antes da cirurgia, os dados correspondentes a este período não tendem a diferir significativamente dos dados obtidos pouco tempo depois do ato cirúrgico, talvez, porque a amplitude de variação dos estados emocionais é pequena. $\mathrm{O}$ mesmo não acontece quando os momentos de avaliação incidem em intervalos de tempo relativamente espaçados entre si, por exemplo, (Momento 1) uma a duas semanas antes da cirurgia, (Momento 2) no pósoperatório de dois anos e (Momento 3) no pós-operatório de cinco anos (Motegi et al., 2003).

Fase Pós-Operatória (a longo-prazo). Depois da cirurgia, os resultados funcionais e estéticos são observados rapidamente e a pessoa encontra segurança emocional para fazer as transformações fundamentais na sua vida. Depreendemos, através da leitura de alguns artigos, que a reparação da aparência facial interfere com a saúde em geral, mas a saúde mental tende a sofrer mais alterações positivas do que a saúde física (Choi et al., 2010; S. Kim et al., 2009).
As mudanças no bem-estar, na qualidade de vida e nas características de personalidade dos pacientes afiguram-se estáveis a longo-prazo, especialmente, dois anos a cinco após a cirurgia. A partir desse período parecem não ocorrer melhorias significativas talvez porque o processo de adaptação à sua transformação ocluso-psicofacial chega ao fim. A pessoa aceitou a nova aparência facial e incorporou as consequências psicossociais na sua personalidade e estilo de vida (Cunningham et al., 2001).

Pogrel e P. Scott (1994) advogam que a intervenção orto-cirúrgica, além de permitir melhorias na saúde física, também constitui um "tratamento" para as perturbações psicológicas, pois os sintomas psicopatológicos quando detectados na fase pré-operatória tendem a diminuir significativamente depois da cirurgia.

Saliente-se que a diminuição dos sintomas psicopatológicos não parece acontecer de uma forma tão abrangente como se supõe. Há evidência de que as perturbações psicopatológicas persistem após a cirurgia por mais cinco anos. É o caso da fobia social, da perturbação dismórfica corporal e da depressão (Finlay et al., 1995; Motegi et al., 2003; C. Phillips et al., 1998; A. Scott et al., 1999), mas não sabemos até que ponto os distúrbios mencionados foram influenciados pela falta de preparação psicológica na fase pré-operatória.

Além do mais, o tipo de discrepância esquelética influencia fortemente o grau de mudanças psicológicas que são experienciadas pelos pacientes. Os resultados empíricos têm patenteado que a transformação é sentida de forma mais intensa e surge mais cedo em pacientes com Classe III de Angle do que em pacientes com Classe II de Angle no que diz respeito à melhoria da auto-atratividade, auto-confiança, insegurança/ preocupação com a aparência e satisfação com o tratamento (Espeland et al., 2008; Gerzanic et al., 2002).

Entre o período pós-operatório de seis meses a dois anos, as mudanças positivas acontecem nos relacionamentos interpessoais, na auto-estima, na auto-confiança, no auto-conceito e continuam presentes após dez a dezasseis anos (Cunningham \& Feinman, 1998; Espeland et al., 2008; Finlay et al., 1995; Hatch et al., 1999; Jacobson, 1984; Lazaridou-Terzoudi et al., 2003; Motegi et al., 2003; A. Scott et al., 2000; Turker et al., 2008).

$\mathrm{O}$ auto-conceito e a auto-estima parecem ser as dimensões psicológicas que demoram mais tempo a melhorar comparativamente à imagem corporal, havendo estudos que reportam o decréscimo dos valores dessas variáveis no pós-operatório de seis a nove meses (Gerzanic et al., 2002; Lee et al., 2008). A este respeito, convém sublinhar que, nos estudos retrospetivos, pedir aos pacientes para recordar o seu percurso de tratamento um ano ou dois anos após a cirurgia pode provocar uma situação de dissonância cognitiva e, por conseguinte, uma tendência para exagerar ou desvalorizar a intensidade das mudanças entre a experiência passada e a atual (LazaridouTerzoudi et al., 2003). Contudo, seria importante, em fu- 
turas investigações, a exploração das narrativas pessoais dos sujeitos relativamente à sua experiência de tratamento, essencialmente, a construção de significados acerca desse processo ao longo do tempo.

Ademais, o conteúdo de alguns questionários utilizados pelos autores, na vertente metodológica, não contém terminologias especificamente relacionadas com a cirurgia ortognática ou até com a preparação ortodôntica. Deste modo, deve haver alguma prudência na interpretação dos resultados quando se diz que, após alguns anos, as mudanças na saúde psicológica ainda se devem à cirurgia ortognática. Na fase da avaliação do pedido pode fazer sentido a aplicação de questionários clínicos (e.g. Symptom Checklist Revised, Orthognathic Quality of Life Questionnaire), mas na fase pós-operatória, os questionários devem abranger escalas que permitem assumir relações estaticamente significativas entre o estatuto psicológico e o tratamento orto-cirúrgico.
De uma forma geral, os pacientes ficam satisfeitos com os resultados da cirurgia ortognática, realizam as suas expectativas e aumentam a sua estabilidade emocional. A taxa de satisfação apresenta-se bastante elevada com valores percentuais entre os $50 \%$ e os $95 \%$ (Jacobson, 1984; S. Kim et al., 2009; Sadek \& Salem, 2007; Turker et al., 2008; R. Williams et al., 2004).

\section{Síntese}

No seguimento da exposição e discussão dos conteúdos abordados, consideramos útil sumariar as principais características psicológicas do paciente analisadas e que podem dificultar ou preludiar o sucesso da intervenção orto-cirúrgica. Na Tabela 3 e a Tabela 4 estão representados alguns exemplos de indicadores em relação aos quais os profissionais de saúde devem estar atentos quando lidam com os pacientes ortognáticos.

Tabela 3

Algumas Características Psicossociais Negativas do Paciente Ortognático

Fase pré-operatória

- Ausência de problema clínico

- Motivação extrínseca

- Historial de distúrbio psiquiátrico ou história familiar de distúrbios afetivos

- Episódios de vida potencialmente traumáticos (bullying)

- Expectativas irrealistas (salvar o casamento, mudar a vida, acabar com o isolamento social)

- Insegurança na tomada de decisão

- Fraco suporte social (membros familiares, amigos que não aceitam a ideia da realização da cirurgia) ou pressão da família

- Distúrbios psicopatológicos (fobia social, ideação suicida, perturbação dismórfica corporal, etc.)

- Inadequadas competências de gestão do stresse

- Não sabe o quer do tratamento ou demonstra-se desinteressado(a)

- Não aceitação das possíveis complicações ou efeitos colaterais da cirurgia

- Realização prévia de cirurgias cosméticas

- Perturbações do sono
Fase pós-operatória (médio e longo-prazo)

- Diminuição da motivação para completar as exigências do tratamento ao nível da higiene, dieta alimentar e ajustamento psicológico

- Sintomatologia depressiva

- Auto-culpabilização inadequada por causa da mudança facial

- Crise de identidade

- Acentuada extroversão (procura de sensações poderá conduzir a comportamentos de risco)

- Insatisfação com os resultados da cirurgia

- Agravamento dos distúrbios psicopatológicos

- Fraco suporte social ou existência de reações negativas dos outros à sua mudança

- Invalidação clinicamente significativa no funcionamento social ou profissional

- Perturbações do sono 
Tabela 4

Algumas Características Psicossociais Positivas do Paciente Ortognático

Fase pré-operatória

- Motivação intrínseca

- Ótimas competências emocionais

- Imagem corporal razoavelmente positiva

- Expectativas realistas quanto aos resultados da cirurgia (a mudança facial será para melhorar o bem-estar psicológico e não para concretizar ambições sociais)

- Expectativas realistas quanto à sintomatologia no pós-operatório imediato

- Sabe o que tem e o que quer do tratamento

- Sente-se preparado(a) para a cirurgia

- Boa rede de suporte (incentivo da família e pessoas significativas)

- Boa capacidade de ajustamento social

- Aceitação das possíveis complicações ou efeitos colaterais da cirurgia
Fase pós-operatória (médio e longo-prazo)

- Satisfação com os resultados da cirurgia

- Mais auto-confiança para interagir com os outros (aumento da assertividade)

- Mais auto-estima

- Estabilidade emocional

- Sente-se bem quando se vê ao espelho, sente-se melhor consigo mesmo(a)

- Atitude menos introvertida (escolhe roupa colorida, sorriso frequente, contato ocular regular, mudança de visual)

- Impacto favorável nos relacionamentos interpessoais (família, amigos e colegas)

- Melhoria da qualidade do sono

- Melhoria da oclusão dentária, respiração e fonação

\section{Conclusão}

O presente artigo procurou oferecer um contributo para o desenvolvimento científico e reflexão acerca da relação entre determinadas características psicossociais dos pacientes e a eficácia do tratamento orto-cirúrgico. Aliás, convenhamos reconhecer que o sucesso da intervenção não é só medido em termos de capacidade da oclusão dentária e melhorias morfológicas, mas também em termos de mudanças no bem-estar psicológico e na qualidade de vida do paciente (Belluci \& Kapp-Simon, 2007). A nosso ver, a cirurgia não muda diretamente a qualidade de vida do paciente, mas permite a segurança emocional para que ele possa fazer importantes mudanças na sua vida. Em concreto, a cirurgia proporciona a melhoria dos estados emocionais ligados à sua imagem corporal que acaba por ter implicações no aumento da auto-estima, auto-confiança, entre outros aspetos. Por seu lado, esta estabilidade emocional traduz-se em perceções e comportamentos positivos que levam o paciente a otimizar o seu bem-estar e qualidade de vida.

No entanto, os profissionais de saúde devem ser proativos no que toca à prevenção do agravamento dos estados emocionais do paciente na fase pré e pós-operatória. Tal como notam Ribas et al. (2005): “a complexidade do tratamento exige uma interação, entre os profissionais e o paciente, de confiança e cumplicidade para se chegar ao objetivo final de satisfação do paciente" (p. 76).

Não obstante os resultados encontrados, estes devem ser interpretados e generalizados com precaução, considerando importante realçar alguns dos seus limites metodológicos. Averiguámos o seguinte: desigualdade de rigor nos critérios de seleção dos participantes; diferenças nas características das amostras (por exemplo, pro- veniência de locais distintos - hospitais, clínicas - o que poderá causar variação nas formas de acesso aos recursos e serviços de saúde, a partir dos quais os pacientes podem ter adquirido perspetivas e experiências diferentes); falta de representatividade e aleatoriedade das amostras; uso de questionários longos e não especificamente aferidos para a população de pacientes ortognáticos; e só alguns estudos recorrem a grupos de controlo.

Os grupos de controlo tornam, de fato, o estudo mais credível, mas cumpre se atente que os resultados variam consoante as características de origem dos grupos (e.g. sujeitos que recusaram a cirurgia ortognática, recusaram qualquer forma de tratamento, fizeram o apenas tratamento ortodôntico ou não possuíam deformidade dentofacial). Tendem a existir diferenças mais acentuadas entre os pacientes ortognáticos e os participantes sem deformidade dentofacial (por exemplo, estudantes universitários).

Estudos futuros devem identificar e caracterizar as fases (ou o processo) de adaptação psicológica do paciente à sua transformação ocluso-psico-facial, de modo a ser possível delimitar os momentos importantes dos períodos de baseline e follow-up.

Consideramos pertinente ainda analisar as diferenças em função do género, idade e tipo de discrepância facial esquelética, uma vez que estas variáveis sócio-demográficas parecem ser as que mais influenciam os resultados empíricos. De um modo geral, a literatura tem apontado que os candidatos mais novos, do género feminino e com Classe II de Angle, tendem a apresentar respostas de ansiedade intensas (Cunningham et al., 2001; A. Scott et al., 1999), são menos felizes com a sua aparência dentofacial (Johnston et al., 2010) e revelam mais dificuldades em se adaptarem à nova mudança facial (S. Kim et al., 2009; Panula et al., 2001). 
Em contraste, o ajustamento apresenta-se mais fácil para o jovem adulto, pois as preocupações relacionadas com a adaptação de papéis sociais na estrutura quotidiana e com a aceitação social parecem ser apaziguadas com os benefícios da cirurgia ortognática (Panula et al., 2001). Os adolescentes, por seu turno, correspondem ao grupo mais vulnerável pois, além das tarefas que caracterizam a construção da identidade e dos próprios desafios emocionais intrínsecos à puberdade, também tendem a ser mais críticos com a sua aparência e menos satisfeitos com o resultado do tratamento orto-cirúrgico do que os restantes grupos (Lazaridou-Terzoudi et al., 2003).

Finalmente, podemos dizer que os avanços no campo da cirurgia ortognática, e a maior compreensão dos aspetos psicossociais dos pacientes que aparecem neste contexto, têm conduzido ao sucesso do tratamento orto-cirúrgico. Todavia, os profissionais de saúde devem estar atentos à presença de sintomas psicopatológicos que refletem, sobretudo, a fobia social, a depressão, a perturbação obsessiva-compulsiva e a perturbação de stresse em pacientes com deformidade dentofacial, porque essa sintomatologia clínica tende a ser mais frequente do que as perturbações psicológicas mais severas, tais como a ideação suicida e a perturbação dismórfica corporal. Através da avaliação e do apoio psicológico e conhecendo, portanto, os fatores protetores e de risco suscetíveis de influenciar o tratamento, será possível ajudar os pacientes a prevenir eventuais sintomas negativos e a sentirem-se melhor preparados para a cirurgia ortognática e suas consequências.

\section{Referências}

Barbosa, M. R. (2008). Contextos relacionais de desenvolvimento e imagem corporal. (Tese de Doutorado nãopublicada). Faculdade de Psicologia e de Ciências da Educação, Universidade do Porto, Portugal.

Belluci, C., \& Kapp-Simon, K. (2007). Psychological considerations in orthognathic surgery. Clinics in Plastic Surgery, 34, 11-16.

Burden, D., Hunt, O., Johnston, C., Stevenson, M., O’Neill, C., \& Hepper, P. (2010). Psychological status of patients referred for orthognathic correction of skeletal II and III discrepancies. Angle Orthodontist, 80(1), 43-48.

Choi, W., Lee, S., McGrath, C., \& Samman, N. (2010). Change in quality of life after combined orthodontic-surgical treatment of dentofacial deformities. Oral Surgery, Oral Medicine, Oral Pathology, Oral Radiology and Endodontics, 109(1), 46-51.

Cunningham, S., \& Feinmann, C. (1998). Psychological assessment of patients requesting orthognathic surgery and the relevance of body dysmorphic disorder. British Journal of Orthodontics, 25(4), 293-298.

Cunningham, S., Gilthorpe, M., \& Hunt, N. (2000). Are orthognathic patients different? European Journal of Orthodontics, 22, 195-202.

Cunningham, S., Gilthorpe, M., \& Hunt, N. (2001). Are pretreatment psychological characteristics influenced by presurgical orthodontics? European Journal of Orthodontics, 23 , 751-758.
Dion, K. K., Berscheid, E., \& Walster, E. (1972). What is beautiful is good. Journal of Personality and Social Psychology, 24, 285-290.

Espeland, L., Høgevold, H., \& Stenvik, A. (2008). A3-year patient-centred follow-up of 516 consecutively treated orthognathic surgery patients. European Journal of Orthodontics, 30, 24-30.

Finlay, P., Atkinson, J. M., \& Moos, A. (1995). Orthognathic surgery: Patient expectations, psychological profile and satisfaction with outcome. British Journal of Oral and Maxillofacial Surgery, 33, 9-14.

Freitas-Magalhães, A. (2007). A Psicologia das Emoções: $O$ fascínio do rosto humano. Porto, Portugal: Edições Universidade Fernando Pessoa.

Gerzanic, L., Jagsch, R., \& Watzke, I. (2002). Psychologic implications of orthognathic surgery in patients with skeletal Class II or Class III malocclusion. International Journal Adult Orthodontics \& Orthognathic Surgery, 17(2), 75-80.

Gonçalves, A., González, M., Vale, T., Moreira, J., Manzanares, C., \& Ustrell, J. (2010). Prevalence of malocclusion and orthodontic treatment need in Braga's children. Poster session presented at the International Association for Dental Research, Barcelona, Spain.

Hatch, J., Rugh, J., Bays, R., Sickels, J., Keeling, S., \& Clark, G. (1999). Psychological function in orthognathic surgical patients before and after bilateral sagittal split ostetomy with rigid and wire fixation. American Journal of Orthodontics and Dentofacial Orthopedics, 115(5), 536-543.

Honigman, R., Phillips, K., \& Castle, D. (2004). A review of psychosocial outcomes for patients seeking cosmetic surgery. Plastic Reconstructive Surgery, 113(4), 229-237.

Hunt, O., Johnston, C., Hepper, P., \& Burden, D. (2001). The psychosocial impact of orthognathic surgery: A systematic review. American Journal of Orthodontics and Dentofacial Orthopedics, 120(5), 490-497.

Jacobson, A. (1984). Psychological aspects of dentofacial esthetics and orthognathic surgery. The Angle Orthodontist, 54(1), 18-35.

Johnston, C., Hunt, O., Burden, D., Stevenson, M., \& Hepper, P. (2010). Self-perception of dentofacial attractiveness among patients requiring orthognathic surgery. Angle Orthodontist, 80(2), 361-365.

Juggins, K., Shute, C., \& Cunningham, S. (2006). Psychological support for orthognathic patients - What do orthodontists want? Journal of Orthodontics, 33, 107-115.

Kim, S., Kim, M., Shin, S., Chun, Y., \& Kim, E. (2009). Evaluation on the psychosocial status of orthognathic surgery patients. Oral Surgery, Oral Medicine, Oral Pathology, Oral Radiology and Endodontics, 108(6), 828-832.

Kiyak, H., McNelll, R., \& West, R. (1985). The emotional impact of orthognathic surgery and conventional orthodontics. American Journal of Orthodontics, 88(3), 224-234.

Lazaridou-Terzoudi, T., Kiyak, H., Athanasiou, A., \& Melsen, B. (2003). Long-term assessment of psychologic outcomes of orthognathic surgery. Journal Oral Maxillofacial Surgery, 61, 545-552.

Lee, S., McGrath, C., \& Samman, N. (2008). Impact of orthognathic surgery on quality of life. Journal Oral Maxillofacial Surgery, 66, 1194-1199.

Leite, P., Camarini, E, Filho, L., Pavan, A., Farah, G., \& Silva, M. (2004). Estudo epidemiológico das deformidades dentofaciais de Maringá. Pesquisa Brasileira Odontopedia Clínica Integrada (João Pessoa), 4(3), 217-220.

Motegi, E., Hatch, J., Rugh, J., \& Yamaguchi, H. (2003). Health- 
related quality of life and psychosocial function 5 years after orthognathic surgery. American Journal of Orthodontics and Dentofacial Orthopedics, 124(2), 138-143.

Nicodemo, D., Pereira, M., \& Ferreira, L. (2007). Cirurgia ortognática: Abordagem psicossocial em pacientes Classe III de Angle submetidos à correção cirúrgica da deformidade dentofacial. Revista Dental Press de Ortodontia e Ortopedia Facial (Maringá), 12(5), 46-54.

Panula, K., Finne, K., \& Oikarinen K. (2001). Incidence of complications and problems related to orthognathic surgery: A review of 655 patients. Journal Oral Maxillofacial Surgery, 59, 1128-1136.

Phillips, C., Bennett, E., \& Broder, H. (1998). Dentofacial disharmony: Psychological status of patients seeking treatment consultation. The Angle Orthodontist, 68(6), 547-556.

Phillips, C., Kiyak, H. A., Bloomquist, D., \& Turvey, T. (2004). Perceptions of recovery and satisfaction in the short term after orthognathic surgery. International Journal Oral Maxillofacial Surgery, 62, 535-544.

Pogrel, M., \& Scott, P. (1994). Is it possible to identify the psychologically "bad risk" orthognathic surgery patient preoperatively? International Journal Adult Orthodontics \& Orthognathic Surgery, 9(2), 105-110.

Ribas, M., Reis, L., França, B., \& Lima, A. (2005). Cirurgia ortognática: Orientações legais aos ortodontistas e cirurgiões bucofaciais. Revista Dental Press de Ortodontia e Ortopedia Facial (Maringá), 10(6), 75-83.

Ryan, F. S., Shute, J., \& Cunningham, S. J. (2009). A Qualitative Study of Orthognathic Patients' Perceptions of Referral to a Mental Health Professional: Part 1 - Questionnaire development. Journal of Orthodontics, 36, 85-92.

Sadek, H., \& Salem, G. (2007). Psychological aspects of orthognathic surgery and its effect on quality of life in Egyptian patients. La Revue de Santé de la Méditerranée Orientale, 13(1), 150-159

Scott, A., Hatch, J., Rugh, J., Rivera, S., Hoffman, T., Dolce, C., et al. (1999). Psychosocial predictors of high-risk patients undergoing orthognathic surgery. International Journal Adult Orthodontics \& Orthognathic Surgery, 14(2), 113-124.

Scott, A., Hatch, J., Rugh, J., Rivera, S., Hoffman, T., Dolce, C., et al. (2000). Psychosocial predictors of satisfaction among orthognathic surgery patients. International Journal Adult Orthodontics \& Orthognathic Surgery, 15(1), 7-15.

Silva, L. M. (2009). Atratividade facial e cirurgia ortognática: Aspetos físicos e emocionais. (Tese de Pós-Graduação em Psicobiologia não-publicada). Faculdade de Filosofia Ciências e Letras, Universidade de São Paulo, Ribeirão Preto, SP.

Stewart, T., \& Sexton, J. (1987). Depression: A possible complication of orthognathic surgery. Journal of Oral and Maxillofacial Surgery, 45, 847-851.

Stirling, J., Latchford, G., Morris, D., Kindelan, J., Spencer, R., \& Bekker, H. (2007). Elective orthognathic treatment decision making: A survey of patient reasons and experiences. Journal of Orthodontics, 34, 113-127.

Turker, N., Varol, A., \& Basa, S. (2008). Perceptions of preoperative expectations and postoperative outcomes from orthognathic surgery: Part I: Turkish female. International Journal Oral Maxillofacial Surgery, 37, 710-715.

Vulink, N., Rosenberg, A., Plooij, J, Koole R, Bergé, S., \& Denys, D. (2008). Body dysmorphic disorder screening in maxillofacial outpatients presenting for orthognathic surgery. International Journal Oral Maxillofacial Surgery, 37, 985-991.

Williams, D., Bentley, R., Cobourne, M., Gibilaro, A., Good,
S., Huppa, C., et al. (2009). Psychological characteristics of women who require orthognathic surgery: Comparison with untreated controls. British Journal of Oral and Maxillofacial Surgery, 47, 191-195.

Williams, R., Travess, H., \& Williams, A. (2004). Patients' experiences after undergoing orthognathic surgery at NHS hospitals in the south west of England. British Journal of Oral and Maxillofacial Surgery, 42, 419-431.

Zane, V., Tavano, L., \& Peres, S. (2002). Avaliação psicológica de pacientes submetidos à cirurgia ortognática. Revista Dental Press de Ortodontia e Ortopedia Facial (Maringá), 7(1), 85-89. 